\title{
Geometrical parameters influence on behavior of the sandwich plates with corrugated core
}

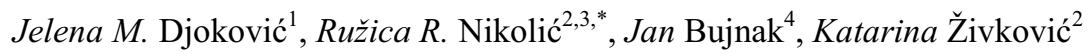 \\ ${ }^{1}$ Technical Faculty in Bor, University of Belgrade, V. Jugoslavije 12, 19210 Bor, Serbia \\ ${ }^{2}$ Faculty of Engineering, University of Kragujevac, Sestre Janjić 6, 34000 Kragujevac, Serbia \\ ${ }^{3}$ Research Center, University of Žilina, Univerzitna 8215/1, 01026 Žilina, Slovakia \\ ${ }^{4}$ Faculty of Civil Engineering, University of Žilina, Univerzitna 8215/1, 01026 Žilina, Slovakia
}

\begin{abstract}
The influence of geometric parameters on behavior and stiffness of sandwich plates with corrugated core is considered in this paper. The following parameters were analyzed: ratio of core sheet and facing sheet thickness and the ratio of the core's pitch to the core's depth, as well as the corrugation angle. It is shown that changes of these parameters can contribute to increase or decrease of the corrugated sandwich plate stiffness.
\end{abstract}

\section{Introduction}

The sandwich plates are the three-layer structures, which consist of the thick core and the two flat plates - faces. In general, case, the faces are made of the highly resistant materials, while the core is made of materials of the lower resistance and density. Due to high resistance of the faces and small mass of the core, one of the most important characteristics of these structures is a good ratio of the structural resistance and mass.

Advantages of the sandwich plates are the high stiffness and resistance, with respect to their mass, good heat and acoustic insulating properties, good aerodynamics, energy absorption for different types of cores, increase of the inner space and simple mounting. Due to those properties, the sandwich structures have wide application in construction industry, aircraft industry, ship building, in the field of the acoustic and heat insulation, the fire protection, etc.

The sandwich plates' core keeps the two separated faces together and stabilizes them by resisting vertical deformations. It enables the whole structure to behave like a thick plate, when the shear resistance is considered. The core also carries the part of the load in the case when the structure is subjected to bending loading. There are several types of core - the foam or the solid core, the honeycomb, prismatic, truss and corrugated core, Vinson [1].

The light and soft materials are usually used for manufacturing the core. For sandwich plates with the core made of foam or in the honeycomb form, all the planar and flexural loads are carried by the faces, while for the sandwich plates with the prismatic and corrugated core, the core also carries the part of the load.

"Corresponding author: ruzicarnikolic@yahoo.com 
Large number of researchers was dealing with analysis of the sandwich structures, subjected to various loadings: Allen [2]; Evans et al. [3]; Brittain et al. [4]; Wicks and Hutchinson [5-6]; Valdevit et al. [7]; Tumino et al. [8] and Cheon and Kim [9]. The most used shape of the core is the honeycomb one, since it possesses the highest stiffness and the shear strength with respect to its mass; however, it requires special attention during the manufacturing. The most studied type of the sandwich plate's failure is wrinkling, which comes to the core's resistance to deformation perpendicular to faces. It is especially prominent with plates whose cores are made of expanded materials. The cores that are made of the highly resistant materials, on the other hand, possess very good resistance to wrinkling, where especially good are the corrugated cores. They are sufficiently stiff to contribute to increase of the flexural stiffness of the whole structure. Unlike the honeycomb cores, the corrugated cores sustain well not only the vertical shear, but the bending and torsion, as well.

Influence of geometrical parameters on behavior of the sandwich plate with the corrugated core, shown in Fig. 1, is analyzed in this paper. The plate's behavior depends on its stiffness. Some geometrical parameters, like ratio of core sheet and facing sheet thickness $\left(t_{c} / t_{f}\right)$, ratio of the core's pitch and the depth $\left(p / h_{c}\right)$, corrugation angle $\alpha$, can contribute to increase or decrease of the stiffness (and thus the resistance) of the plate with the corrugated core.

\section{Corrugated plate geometrical characteristics and stiffness}

The sandwich plate with the corrugated core, Fig. 1, consists of two flat plates - faces of thickness $t_{f}$ and the corrugated core sheet thickness $t_{c}$. The dimensions of the plate are as shown in Fig. 1: $\alpha$-the corrugation angle, $h_{c}$ - the core's depth measured from the center line at crest to center line at trough, $h$ - distance between the middle planes of the face sheets, $2 p$ - the face sheets and $f$ - the length of corrugation flat segment. Subscripts $c$ and $f$ refer to core's and the faces' properties, respectively.

In analysis of the sandwich plates with the corrugated core, for the case of flexural loading, the following assumptions were made: the analyzed corrugated core is symmetric, the faces are made of the same material and with the same thickness, the core material is isotropic-elastic, the sandwich plate's deformations are small, the plate's thickness is significantly smaller than the core's depth, the core influences the flexural stiffness in the $x$ direction but not in the $y$-direction, the plate is infinitely long in the $z$-direction, the core can sustain the lateral shear stress and it also affects the flexural and axial stiffnesses, while the lateral shortening of the core is not taken into account.

The flexural stiffnesses in the $x$ and $y$ directions are determined as, Libove and Hubka [10]:

$$
B_{x}=2 E_{f} I_{f}+E_{c} I_{c}, \quad B_{y}=\frac{E_{f} I_{f}}{1-v_{f}^{2}\left(\frac{E_{f} I_{f}}{B_{x}}\right)},
$$

respectively, where $E_{c}$ and $v_{c}$ are the Young modulus of elasticity and the Poisson's ratio of the core's material, respectively, while $E_{f}$ and $v_{f}$ are the same characteristics for the faces' material. 

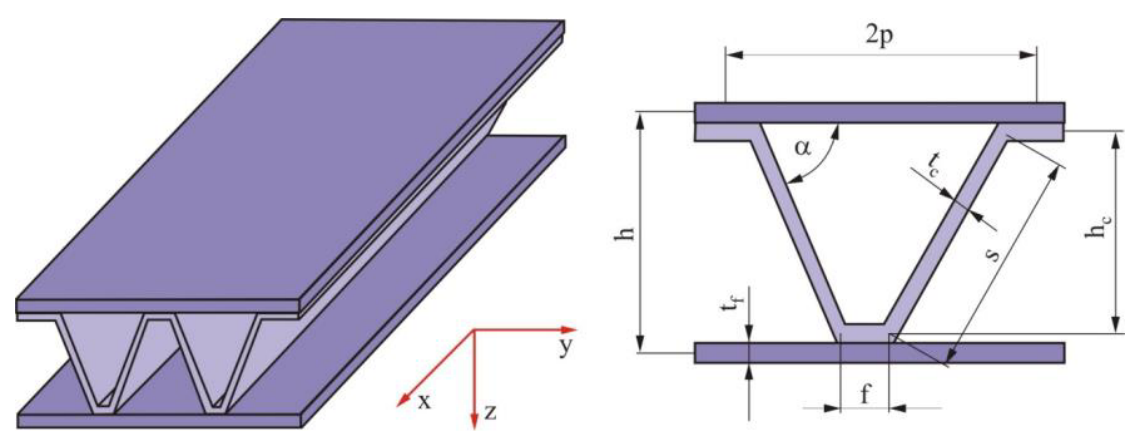

Fig. 1. The geometry of the sandwich plate with the corrugated core.

The cross-section's moment of inertia of the core, parallel to the $y z$ plane, per unit width, taken for the symmetry axis of the cross section, $I_{c}$, and moment of inertia of the face, considered as the membrane, with respect to the middle plane of the sandwich plate, $I_{f}$, respectively are given by:

$$
I_{f}=2 p \cdot t_{f} \cdot\left(\frac{h_{c}}{2}\right)^{2}, I_{c}=\frac{f \cdot t_{c} \cdot h_{c}^{2}}{2}+\frac{2 \cdot t_{c} \cdot h_{c}^{3}}{12 \cdot \sin \alpha} .
$$

The torsional stiffness is determined as:

$$
B_{x y}=2 G_{f} I_{f}
$$

where $G_{f}$ is the shear modulus of the face.

\section{Results and discussion}

In Figs. 2, 3 and 4 are presented variations of the sandwich plate's stiffnesses $B_{x}, B_{y}$ and $B_{x y}$ in terms of the $\left(t_{c} / t_{f}\right)$ ratio, the core angle $\alpha$ and the $\left(p / h_{c}\right)$ ratio, obtained according to equations (1) to (3), by the programming package Mathematica ${ }^{\circledR}$. The case considered is that of the rectangular sandwich plate, loaded by the distributed load $q=10 \mathrm{kN} / \mathrm{m}^{2}$. The plate is simply supported along the edges, which means that the boundary conditions are: for $x=$ const. the deflection is $w=0$, the bending moment is $M_{x}=0$, as well as the transversal force $Q_{y}=0$. The face and the core materials are identical with the elastic characteristics: $E=208 \mathrm{GPa}$ and $v=0.3$.

In Fig. 2 is shown influence of variation of the of core sheet and facing sheet thickness ratio on flexural stiffnesses $B_{x}$ and $B_{y}$, as well as on the torsional stiffness $B_{x y}$, for the case of the corrugation angle $\alpha=60^{\circ}$, the core's pitch to depth ratio $\left(p / h_{c}\right)=1$ and for the core's depth to core thickness ratio $\left(h_{c} / t_{c}\right)=10$. 


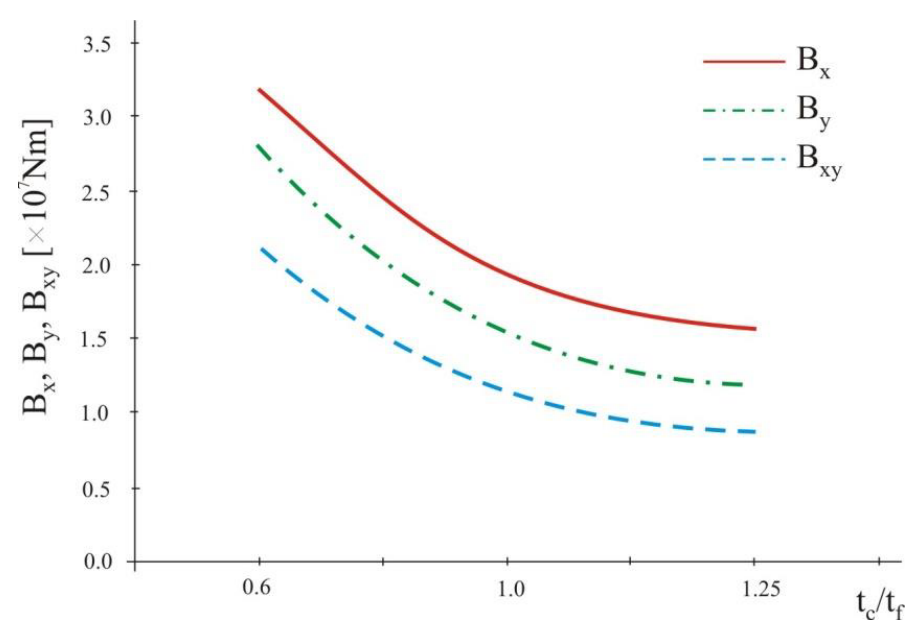

Fig. 2. Variation of the flexural and torsional stiffnesses of the sandwich plate in terms of the $\left(t_{c} / t_{f}\right)$ ratio.

From Fig. 2 one can see that all the three stiffnesses are decreasing with the face thickness to the core depth ratio increase, for the same values of the corrugation angle $\alpha$ and the ratios $\left(p / h_{c}\right)$ and $\left(h_{c} / t_{c}\right)$. As the $\left(t_{c} / t_{f}\right)$ ratio increases, for the certain value of the core's sheet thickness $t_{c}$, the facing sheet thickness $t_{f}$ decreases, what causes the lower stiffness of the corrugated sandwich plate, since the faces carry larger portion of the bending loading. If the tendency of the stiffnesses decrease is compared to tendency of the $\left(t_{c} / t_{f}\right)$ ratio increase, one can notice that for the $\left(t_{c} / t_{f}\right)$ increase from 0.6 to 1.0 , the flexural stiffnesses $B_{x}$ and $B_{y}$ decrease for about $45 \%$, while the torsional stiffness $B_{x y}$ decrease for about $40 \%$. The same trend is continued for the $\left(t_{c} / t_{f}\right)$ ratio increase from 1.0 to 1.25 , where $B_{x}$ and $B_{y}$ decrease for about $22 \%$, while the torsional stiffness $B_{x y}$ decrease for about $17 \%$. This leads to conclusion that the $\left(t_{c} / t_{f}\right)$ ratio has stronger influence on the flexural stiffnesses than on the torsional one.

In Fig. 3 is shown the influence of the corrugation angle $\alpha$ variation on the flexural and the torsional stiffnesses, for the following geometrical parameters of the sandwich plate: the core sheet and facing sheet thickness ratio $\left(t_{c} / t_{f}\right)=1$, the core pitch to depth's ratio $\left(p / h_{c}\right)=1$ and the core's depth to core thickness ratio $\left(h_{c} / t_{c}\right)=10$.

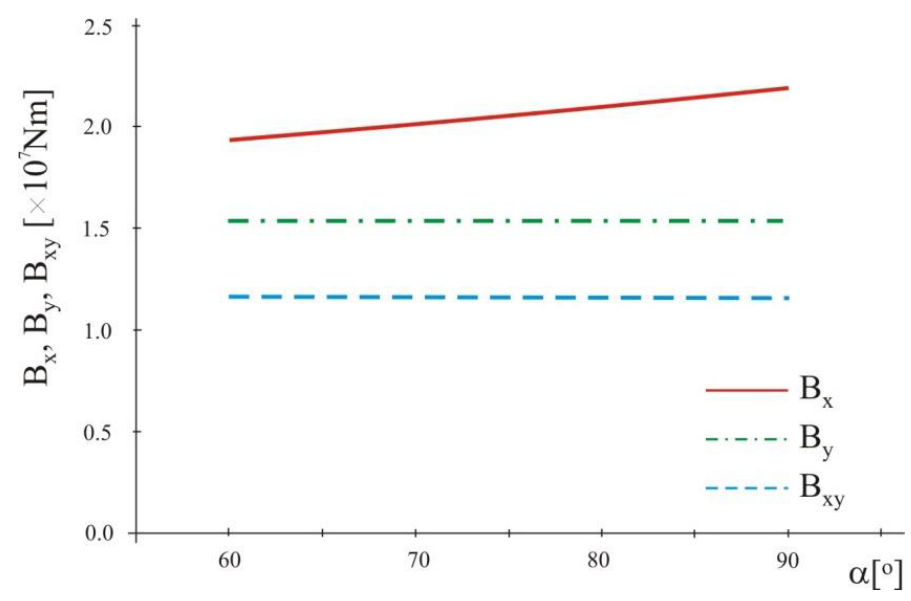

Fig. 3. Variation of the sandwich plate stiffnesses in terms of thecorrugation angle $\alpha$. 
From Fig. 3 could be noticed that flexural stiffness $B_{x}$ increases with increase of the corrugation angle $\alpha$, while the stiffnesses $B_{y}$ and $B_{x y}$ remain constant, for the same values of ratios: $\left(t_{c} / t_{f}\right),\left(p / h_{c}\right)$ and $\left(h_{c} / t_{c}\right)$.

In Fig. 4 is presented the influence of the core's pitch to depth ratio $\left(p / h_{c}\right)$ on flexural stiffnesses $B_{x}$ and $B_{y}$ and on the torsional stiffness $B_{x y}$, for the following values of the geometrical parameters of the sandwich plate: $\alpha=60^{\circ},\left(t_{c} / t_{f}\right)=1$ and $\left(h_{c} / t_{c}\right)=10$.

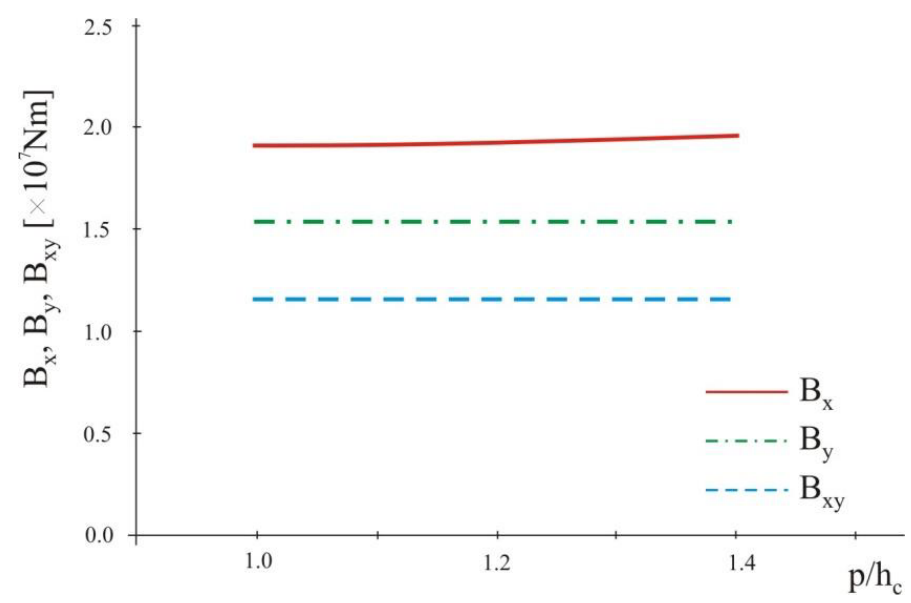

Fig. 4. Variation of the sandwich plate stiffnesses in terms of the $\left(p / h_{c}\right)$ ratio.

From Fig. 4 one can notice that the flexural stiffness $B_{x}$ slightly increases with increase of the core's pitch to depth ratio, while the stiffnesses $B_{y}$ and $B_{x y}$ remain constant, for the same values of the corrugation angle $\alpha$ and ratios $\left(t_{c} / t_{f}\right),\left(p / h_{c}\right)$ and $\left(h_{c} / t_{c}\right)$.

\section{Conclusion}

The influence of the geometrical parameters of a sandwich plate with the corrugated core on its behavior was considered in this paper. The following parameters' influences were analyzed: ratio of the core sheet thickness to the sandwich plate face's thickness, the corrugation angle and the ratio of the core's pitch to depth.

Based on results obtained from that analysis, one can notice that all the stiffnesses of the sandwich plate (two flexural and a torsional) are decreasing with increase of the core sheet thickness to the face's thickness ratio, though the influence is more prominent for the flexural stiffnesses than for the torsional one. As that ratio increases, for the constant value of the core sheet thickness, the face's thickness decreases, what causes the decrease of the sandwich plate's flexural stiffness, since for the corrugated plates the faces are carrying the larger portion of the bending loading.

For the case of the corrugation angle increase and increase of the core's pitch to depth ratio, the main flexural stiffness increases slightly, while the one in the perpendicular direction and the torsional stiffness remain constant.

Based on analysis that was conducted within this research, certain recommendation can be given for the selection of the geometrical parameters of the sandwich plates with the corrugated core. It is shown that the lower values of the investigated parameters make the sandwich plate more resistant. It would be the best to choose the plate's parameters in such a way that face and core sheet thicknesses would be the same, $\left(t_{f}=t_{c}\right)$, that the corrugation angle value would be about $60^{\circ}$ and that the value of the core's pitch to depth ratio $\left(p / h_{c}\right)$ would be between 1 and 1.2. 
This research was partially supported by the Ministry of Education, Science and Technological Development of Republic of Serbia through Grants ON174001, ON174004 and TR32036 and by European regional development fund and Slovak state budget by the project "Research Center of the University of Žilina" - ITMS 26220220183. The authors are very grateful for this funding.

\section{References}

1. J.R. Vinson, Appl. Mech. Rev. 1. 54, 201-214 (2001)

2. H.G. Allen, Analysis and Design of Structural Sandwich Panels (Pergamon Press, Oxford, 1969).

3. A.G. Evans, J.W. Hutchinson, M.F. Ashby, Prog. Mater. Sci 43(3), 171-221 (1998)

4. S.T. Brittain, Y. Sugimura, J.A. Schueller, A.G. Evans, G.M. Whitesides, J. Microelectromech. Syst. 10, 113-120 (2001)

5. N. Wicks and J.W. Hutchinson, Int. J. Sol. Struct. 38, 5165-5183 (2001)

6. N. Wicks and J.W. Hutchinson, Mech. Mater. 36, 739-751 (2004)

7. L. Valdevit, J.W. Hutchinson, A.G. Evans, Int. J. Sol. Struct. 41, 5105-5124 (2004)

8. D. Tumino, T. Ingrassia, V. Nigrelli, G. Pitarresi, V. Urso-Miano, Frat. Integr. Strut. 30, 317-326 (2014)

9. Y.-J.Cheon, H.-G. Kim, J. Mech. Sci. Techn. 29(3), 1217-1223 (2015)

10. C. Libove, R.E. Hubka, Elastic constants for corrugated core sandwich plates, Techn. Note 2289 (National Advisory Committee for Aeronautics, Washington, 1951) 